Biochimica et Biophysica Acta, 587 (1979) 75-88

(C) Elsevier/North-Holland Biomedical Press

BBA 29053

\title{
COMPOSITION OF THE AQUEOUS PHASE OF CHROMAFFIN GRANULES
}

\author{
ROMITA SEN ${ }^{\text {a }}$, ROBERT R. SHARP a , LAWRENCE E. DOMINO ${ }^{a}$ and \\ EDWARD F. DOMINO ${ }^{b}$ \\ a Department of Chemistry, The University of Michigan, Ann Arbor, MI 48109 and \\ b Department of Pharmacology, The University of Michigan, Ann Arbor, MI 48109 (U.S.A.)
}

(Received January 12th, 1979)

Key words: Chromaffin granule; Catecholamine; Aqueous phase; NMR; (Adrenal medulla)

\section{Summary}

Nuclear magnetic resonance spectroscopy has been used to determine the composition of the aqueous phase of bovine chromaffin granules. Relative concentrations of catecholamines (epinephrine plus norepinephrine), ATP and chromogranins have been measured from integrated intensities in the proton spectra using computer simulation techniques. Most or all of the catecholamines $(97 \pm 8 \%)$ are present in the aqueous phase and contribute to the high resolution spectrum. The catecholamine:ATP molar ratio $(4.41 \pm 0.45)$ determined by NMR is close to the value (4.45) derived from biochemical assay indicating that most or all of the ATP is present with catecholamine in the aqueous phase. Catecholamine:protein ratios show that approximately $45 \%$ of the soluble protein freed by lysis is not NMR visible. Intensity from this fraction does not appear under highly denaturing conditions ( $8 \mathrm{M}$ urea) but reappears after hydrolysis. This behavior is similar to that of recently isolated soluble lipoprotein complexes. Variations in the NMR spectra associated with (1) different preparative procedures; (2) different suspension media, and (3) increasing osmolality are described. The fact that high concentrations of epinephrine and ATP (approximately $700 \mathrm{mM}$ total) are dissolved in the aqueous phase implies that solution phase interactions at least partially ionic in nature are responsible for the low internal osmolality of chromaffin granules in vivo. Ordered phases containing a substantial fraction of the total catecholamine in an osmotically inactive form are not present. 


\section{Introduction}

Chromaffin granules, which are the site of catecholamine storage in the adrenal medulla, maintain high concentrations of catecholamines and ATP in an aqueous medium, the osmolality of which is much lower than the concentrations of these components would suggest [1]. Although the biochemical composition of chromaffin granules has been studied extensively [2-5] many structural features of the internal medium remain unclear. It has been demonstrated by nuclear magnetic resonance (NMR) spectroscopy that a substantial portion of the soluble components, including most of the catecholamines, are present in an aqueous solution phase [6-11]. These results agree with density $[12,13]$ and light-scattering [14] measurements indicating that the intragranular fluid is in osmotic equilibrium with the external medium. The internal fluid apparently contains a substantial quantity of osmotically inactive material with an undetermined catecholamine content [13-15]. The nature of the interactions between the soluble components that leads to a lowering of the internal osmolality has still not conclusively been demonstrated, although it has been the subject of considerable debate [16-23].

Pertinent to this question are several reports of two or more physically distinct intragranular pools of catecholamine [18,24-28]. Support for this hypothesis has been based on the observation of biphasic efflux kinetics from chromaffin granules incubated at $37^{\circ} \mathrm{C}[24,25]$, the non-stoichiometric concentrations of catecholamines and ATP [26], and on the existence of bound catecholamine fractions that sediment with the membrane $[27,28]$. Of course highresolution NMR spectra detect only molecules dissolved in mobile, essentially isotropic phases. Catecholamines that are membrane bound, or associated with precipitates, globular proteins or high molecular weight aggregates would probably not give resolved resonances. An exception to this statement could occur if catecholamines were exchanging rapidly $(\tau \approx 10 \mathrm{~ms})$ between bound and free sites. Chemical exchange on the NMR time scale would cause pronounced line broadening however, and in some circumstances could be demonstrated if present [29].

In this paper we report quantitative measurements of the composition of the NMR-visible aqueous phase. The composition is compared to the total concentrations of soluble components measured by biochemical assay. The results indicate that most, if not all, of the catecholamines and ATP are dissolved in the aqueous phase. Upper limits to the NMR-invisible fractions have been obtained. Approximately $45 \%$ of the soluble protein that is released upon lysis is not NMR visible. Spectral variations found in samples prepared by two different methods (differential centrifugation in $0.32 \mathrm{M}$ sucrose [32] and centrifugation on a Ficoll-sucrose- ${ }^{2} \mathrm{H}_{2} \mathrm{O}$ gradient [31]) and suspended in electrolyte solutions of varying osmolality are described.

\section{Experimental procedures}

Preparation of chromaffin granules for NMR study. Chromaffin granules were isolated from bovine adrenal glands. A 'large granule fraction' was prepared using the procedure of Bartlett and Smith [30]. Further purification of 
this fraction was carried out in one of two ways: the first using a Ficoll-sucrose${ }^{2} \mathrm{H}_{2} \mathrm{O}$ gradient [31], and the second by differential centrifugation at $35000 \times \mathrm{g}$ for $20 \mathrm{~min}$ [32]. The pelleted granules obtained by each method were washed twice with solutions of $\mathrm{K}_{2} \mathrm{SO}_{4}(0.15 \mathrm{M}$ and $0.16 \mathrm{M})$ or $\mathrm{KCl}(0.16 \mathrm{M}$ and 0.20 M) in ${ }^{2} \mathrm{H}_{2} \mathrm{O}$. These washings removed most of the sucrose and reduced the concentration of protons from $\mathrm{H}_{2} \mathrm{O}$ in the chromaffin granule pellet. The NMR spectra recorded were of chromaffin granules suspended in the same $\mathrm{K}_{2} \mathrm{SO}_{4}$ and $\mathrm{KCl}$ solutions used to wash the pellet and contained approximately $0.25 \mathrm{~g}$ wet pellet/ml of suspension. Lysis was monitored directly by NMR, making use of the chemical shift between the aromatic peaks of free and vesicle-bound catecholamine (see below). Preparations used for spectral fitting were essentially free from lysis.

Preparation of lysate for NMR study. A weighed quantity of chromaffin granule pellet was lysed by placing it in 20 vols. of ice-cold ${ }^{2} \mathrm{H}_{2} \mathrm{O}$. The solution was frozen, thawed and centrifuged at $100000 \times \mathrm{g}$ for $60 \mathrm{~min}$ at $2^{\circ} \mathrm{C}$ in a Beckman L5-50 ultracentrifuge. The supernatant was removed carefully and kept on ice. Another 20 vols. of ${ }^{2} \mathrm{H}_{2} \mathrm{O}$ were added to the sediment in the ultracentrifuge tube and after an identical series of steps the supernatant obtained was combined with the supernatant from the first lysis. The combined supernatant, referred to as the 'soluble lysate', was observed directly on the NMR or concentrated by ultrafiltration before study.

$N M R$ data. All spectra were recorded at $3-5^{\circ} \mathrm{C}$. Fourier transform 100 $\mathrm{MHz}$ proton spectra were obtained on a JEOL JNM/PFT-100 spectrometer. Peak integrals were computed digitally between specified frequency limits by the EC-100 data system. Spectra were obtained using a $90^{\circ}$ flip angle and a delay of $1.5 \mathrm{~s}$. This delay is greater than $4 T_{1}$ for all peaks in the spectrum.

Assays. Protein was assayed by the Lowry method [33] or by a dye-binding technique [34]. When using the Lowry test, the protein was precipitated using trichloroacetic acid to eliminate interference from catecholamines. Bovine serum albumin was used as the standard in the Lowry method and $\gamma$-globulin for the dye-binding method. The two protein assays were checked against each other for solutions of purified chromogranins and found to give comparable results.

Catecholamines were assayed by the trihydroxyindole method [35] using a Perkin Elmer MPF-44A spectrofluorometer.

Determination of concentrations of protein, catecholamines and ATP from $N M R$ spectra. A computer fit of the integrated intensity over a sequence of frequency intervals of the high resolution ${ }^{1} \mathrm{H}$ NMR spectrum was carried out. A non-linear least-squares program supplied by the University of Michigan Statistics Laboratory [36] was used to fit the NMR integrals with the concentration ratios as adjustable parameters. The steps involved were as follows:

(i) Integrals were measured across the spectrum over regions typically 20 $\mathrm{Hz}$ wide. The regions containing $\mathrm{H}^{2} \mathrm{HO}$ and sucrose peaks were not included in the fit.

(ii) The experimental spectrum was simulated assuming Lorenzian line shapes. The peak frequencies and linewidths were measured experimentally in the case of catecholamines and ATP, and the frequencies were taken from the literature [8] for the soluble proteins. All protein linewidths were assumed 
equal and were estimated from trial visual fits of experimental and simulated spectra.

(iii) Since the integrated intensity of the peaks is proportional to the concentrations of the species contributing to that peak, the experimental integral in any region, $i$, of the spectrum can be expressed as

$I_{\mathrm{i}}=a \sum_{\mathrm{j}} c_{\mathrm{j}} A_{\mathrm{ij}}$

where $c_{\mathrm{i}}$ is the concentration of the $j^{\text {th }}$ molecule, and $A_{\mathrm{ij}}$ is the contribution to the integral in the $i^{\text {th }}$ region from the $j^{\text {th }}$ molecule when its concentration is unity (computed from simulated spectra). Assuming a Lorenzian line shape,

$$
A_{\mathrm{ij}}=a \sum_{\substack{\mathbf{k} \\ \text { peaks }}} \frac{A_{\mathrm{kj}}}{1+T_{2 \mathbf{k}}^{2}\left(\omega-\omega_{\mathrm{k}}\right)^{2}}
$$

where $A_{\mathrm{kj}}=c_{\mathrm{j}} n_{\mathrm{k}} ; n_{\mathrm{k}}$ is the number of protons in the $k^{\text {th }}$ site; $T_{2 \mathrm{k}}$ is the spinspin relaxation time of the $k^{\text {th }}$ site, and $\omega$ is the angular frequency $(=2 \pi \nu)$. $T_{2 \mathrm{k}}=\left(\pi \Delta v_{1 / 2, \mathrm{k}}\right)^{-1}$ where $\Delta \nu_{1 / 2, \mathrm{k}}$ is the linewidth of the $k^{\text {th }}$ site. The constant, $a$, normalizes experimental integrals to those derived from simulated spectra and is obtained from the fitting procedure. A value for $A_{\mathrm{ij}}$ can be calculated in any region of the spectrum lying between two values of $\nu$, if the peak positions, $\nu_{\mathrm{k}}$, the number of protons contributing to a peak and the linewidths, $\Delta \nu_{1 / 2}$, are known.

In the NMR analysis carried out for the results presented here, the high resolution spectrum of chromaffin granules was assumed to be due to ATP, catecholamines (CA), and chromogranin A. The amino acid composition of the total soluble proteins is very similar to that of chromogranin $A[2]$.

From Eqn. 1

$$
\begin{aligned}
& I_{\mathrm{i}}=a\left[c_{\mathrm{CA}} A_{\mathrm{CA}, \mathrm{i}}+c_{\mathrm{ATP}} A_{\mathrm{ATP}, \mathrm{i}}+c_{\text {protein }} A_{\text {protein }, \mathrm{i}}\right] \\
& \quad=a c_{\mathrm{CA}}\left[A_{\mathrm{CA}, \mathrm{i}}+\frac{c_{\mathrm{ATP}}}{c_{\mathrm{CA}}} \frac{A_{\mathrm{ATP}, \mathrm{i}}}{A_{\mathrm{CA}, \mathrm{i}}}+\frac{c_{\text {protein }}}{c_{\mathrm{CA}}} \frac{A_{\text {protein }, \mathrm{i}}}{A_{\mathrm{CA}, \mathrm{i}}}\right] \\
& \text { or } y=\theta_{1}\left(x_{1}+\theta_{2} x_{2}+\theta_{3} x_{3}\right)
\end{aligned}
$$

A non-linear least-squares fit of this function to the data values, $x_{1}, x_{2}, x_{3}$ and $y$ by iterations on the parameters $\theta_{1}, \theta_{2}$ and $\theta_{3}$ was carried out using the program, BMDX85 [36]. $\theta_{1}$ normalizes the integrals from experimental and simulated spectra. A fit of the non-linear form (Eqn. 2) rather than the linear form (Eqn. 1) was used in order to obtain standard deviations for the concentration ratios, $\theta_{2}$ and $\theta_{3}$.

The absolute concentration of catecholamines was determined by comparing the integral of the aromatic catecholamine peak with that of the methyl peak of the sodium salt of 3-(trimethylsilyl)propanesulfonic acid (TSS), which is well separated to high field of the chromaffin granule spectrum.

\section{Results}

NMR spectra of chromaffin granule suspensions

High resolution proton spectra of chromaffin granules prepared according to 


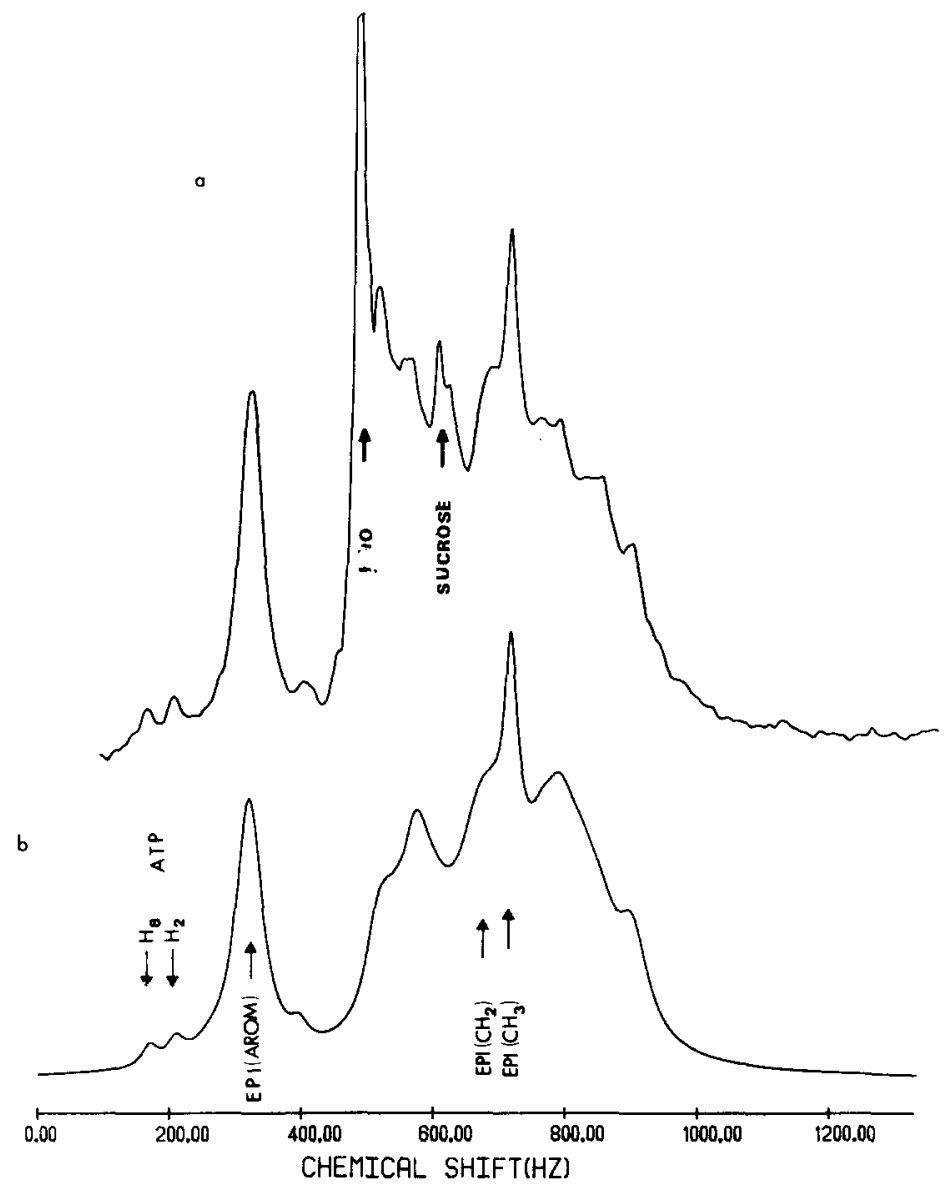

Fig. 1. (a) Proton spectrum of chromaffin granules prepared by differential centrifugation through 0.32 $M$ sucrose and suspended in $0.15 \mathrm{M} \mathrm{K}_{2} \mathrm{SO}_{4} / /^{2} \mathrm{H}_{2} \mathrm{O}\left(4^{\circ} \mathrm{C}\right), 32$ scans. Solvent peak is decoupled. (b) Computer simulation of the spectrum. Chemical shifts are given on the $\tau$ scale, where the methyl peak of TSS lies at $1000 \mathrm{~Hz}$. EPI, epinephrine.

procedures explained in Experimental procedures were obtained. A typical spectrum of granules prepared by differential centrifugation in $0.32 \mathrm{M}$ sucrose and suspended in $\mathrm{K}_{2} \mathrm{SO}_{4}(337$ mosM) is shown with the computer simulation in Fig. 1. In all spectra the characteristic peaks of ATP, catecholamines and chromogranins are present. The linewidths of the resonances differ, however, depending on the suspension medium and the method of preparation. It was observed that:

(1) Chromaffin granules purified on a Ficoll-sucrose $-{ }^{2} \mathrm{H}_{2} \mathrm{O}$ gradient give broader peaks than those prepared by differential centrifugation in $0.32 \mathrm{M}$ sucrose. Examples of the broadening seen in the region downfield of $\mathrm{H}^{2} \mathrm{HO}$ are shown in Fig. 2. The ATP peaks (H-2 and H-8) are essentially unresolved in Ficoll preparations while epinephrine peaks are broadened by about $30 \%$. This finding is consistent with a previous report that the morphological characteristics of granules prepared on a Ficoll gradient are somewhat different from granules prepared by differential centrifugation [37]. 


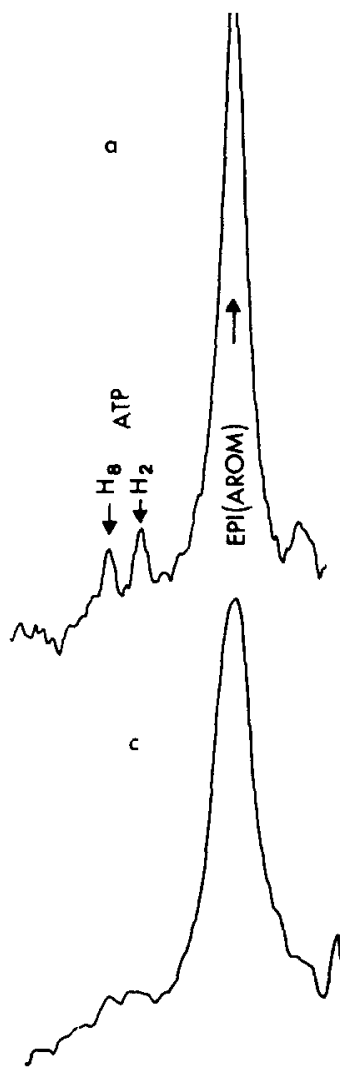

$200 \mathrm{~Hz}$

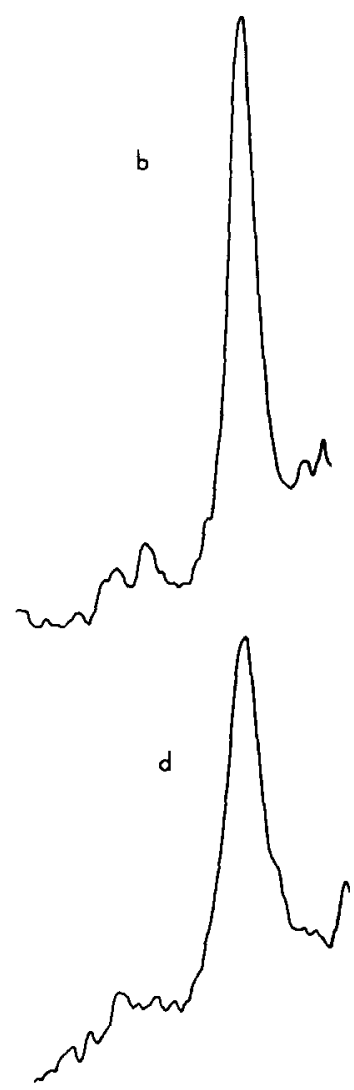

$200 \mathrm{~Hz}$

Fig. 2. Chromaffin granule spectra $\left(4^{\circ} \mathrm{C}\right.$ ) of the region downfield of the $\mathrm{H}^{2} \mathrm{HO}$ peak; (a) differential centrifugation in $0.32 \mathrm{M}$ sucrose, washed and suspended in $0.15 \mathrm{M} \mathrm{K}_{2} \mathrm{SO}_{4}, 32$ scans; (b) same, but washed and suspended in $0.16 \mathrm{M} \mathrm{KCl}$; (c) Ficoll-sucrose $-2 \mathrm{H}_{2} \mathrm{O}$ gradient, washed and suspended in $0.15 \mathrm{M} \mathrm{K}_{2} \mathrm{SO}_{4}$, 61 scans; (d) same as for (c), but washed and suspended in 0.16 M KCI. EPI, epinephrine.

(2) Chromaffin granules suspended in $\mathrm{K}_{2} \mathrm{SO}_{4}$ show broader lines as the osmolality is increased as would be expected from shrinkage of granules. As an example, the spectrum in Fig. 3a (chromaffin granules suspended in 360 mosM $\mathrm{K}_{2} \mathrm{SO}_{4}$ ) may be compared with that in Fig. 1a (339 mosM $\left.\mathrm{K}_{2} \mathrm{SO}_{4}\right)$. Increasing osmolality with $\mathrm{KCl}$ as supporting electrolyte shows a similar effect.

(3) Significant shifts and/or gross selective broadening are seen in the methylene peak of catecholamines in granules suspended in $\mathrm{K}_{2} \mathrm{SO}_{4}$ solutions of 360 or greater osmolality. This effect is illustrated by comparing Fig. 1a (337 mosM), which contains a broad but well-defined methylene resonance, with Fig. 3a (360 mosM), in which the methylene peak is not visible. This effect, which was observed repeatedly in $360 \operatorname{mosM~} \mathrm{K}_{2} \mathrm{SO}_{4}$, has not been observed in other suspension media $[6-10]$.

Mitochondria and lysosomes are present as impurities in the chromaffin granule preparations, but they do not give interfering structure in the high resolution proton NMR spectra. This was confirmed by observing the mito- 


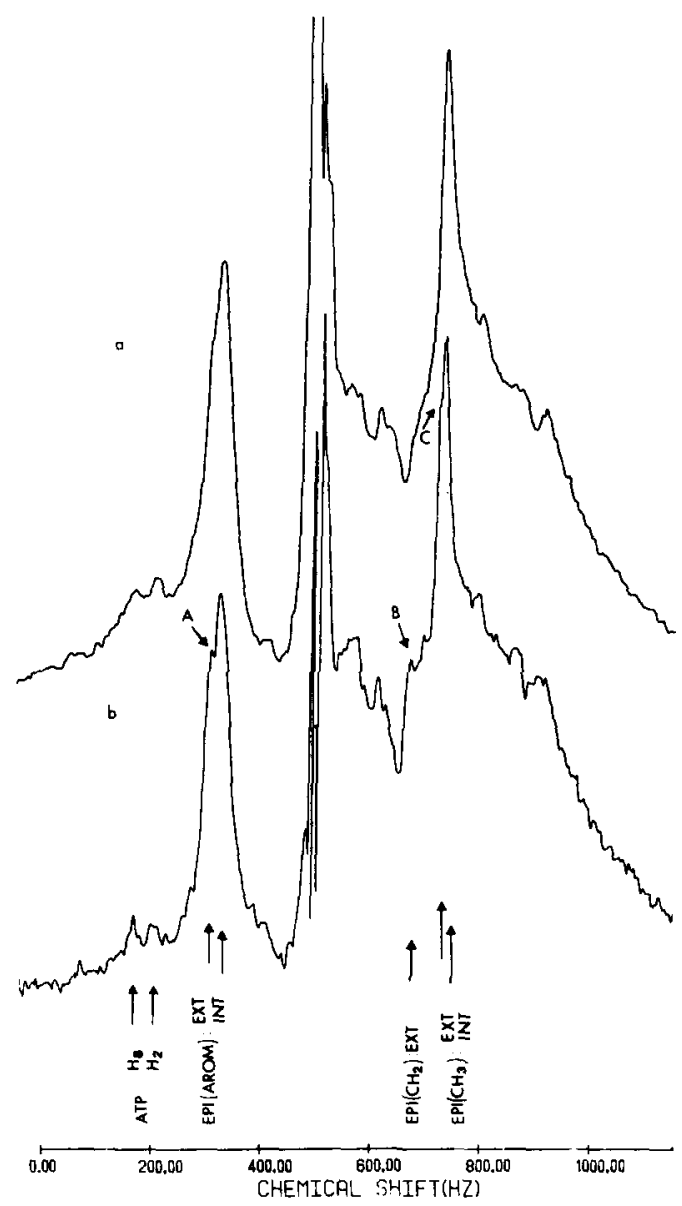

Fig. 3. Effects of osmolality and lysis on spectra $\left(4^{\circ} \mathrm{C}\right)$ of chromaffin granules prepared by centrifugation through $0.32 \mathrm{M}$ sucrose, washed and suspended in $0.16 \mathrm{M} \mathrm{K}_{2} \mathrm{SO}_{4}$. (a) Spectrum taken $2.5 \mathrm{~h}$ after final washing; (b) same sample, spun down at $12 \mathrm{~h}$, spectrum taken $24 \mathrm{~h}$ after resuspension (100 scans). Peaks $A-C$ arise from the aromatic, methylene and methyl resonances of free catecholamine. EPI, epinephrine.

chondrial fraction from the Ficoll-sucrose- ${ }^{2} \mathrm{H}_{2} \mathrm{O}$ gradient, which gave no peaks except a low intensity very broad resonance in the protein region.

\section{Stability of the samples}

The stability of the various suspensions used was checked by NMR. Fig. 3 contains spectra taken of chromaffin granules prepared by differential centrifugation in $0.32 \mathrm{M}$ sucrose followed by washes with $0.16 \mathrm{M} \mathrm{K}_{2} \mathrm{SO}_{4}$ and resuspension in the same solution. The spectra show no change for several hours after preparation (Fig. 3a). If the sample is left overnight at $5^{\circ} \mathrm{C}$, the spectrum shows peaks due to free catecholamines and ATP to the low field side of the main peaks. These peaks can be removed if the suspension is centrifuged, the supernatant discarded and the granules resuspended. Fig. $3 \mathrm{~b}$ shows the same chromaffin granule suspension $36 \mathrm{~h}$ after preparation. The peaks due to lysed material are clearly seen, the positions correspond to those from chromaffin 
granules lysed by hyposmotic shock. The appearance of separate peaks due to internal and external catecholamine provides a direct NMR method for monitoring lysis. The shift in the aromatic region has been noted previously [20].

\section{Composition of NMR-visible interior of chromaffin granules}

Several different preparations of chromaffin granules were studied to determine the NMR-visible concentrations of ATP, catecholamines and proteins.

Table I summarizes the data from biochemical assays of protein and catecholamines. The concentrations are the average of two sets of experiments. The catecholamine : protein ratios found agree closely in most cases with values reported in the literature [2]. Values from the Ficoll-sucrose- ${ }^{2} \mathrm{H}_{2} \mathrm{O}$ preparations that were washed in $\mathrm{KCl}$ were somewhat low due to increased fragility of the granules. On an average $67 \%$ of the total protein in chromaffin granules was present in the soluble fraction. With the lysis procedure used, essentially all of the catecholamines were recovered in the soluble fraction; less than $0.2 \%$ of catecholamines sedimented with the membrane fraction. NMR spectra of chromaffin granules contain peaks only from those molecules in a highly mobile state, that is, in the solution phase. The catecholamine to protein ratio seen by NMR should, therefore, correspond to values in column 5 of Table I, namely g soluble catecholamine:g soluble protein.

Table II contains the ratios of catecholamine: protein and catecholamine: ATP as determined by NMR. Fig. $1 \mathrm{~b}$ is typical of a spectrum simulated by the fitting procedure. The values reported are an average of the number of determinations indicated. For each determination between 20 and 30 regions, each about $20 \mathrm{~Hz}$ in width, were fitted. Error limits were calculated at the $95 \%$

TABLE I

BIOCHEMICAL ASSAYS OF PREPARATIONS USED IN THIS STUDY

\begin{tabular}{|c|c|c|c|c|c|c|}
\hline \multirow[t]{2}{*}{ Preparation of sample } & \multicolumn{2}{|c|}{$\begin{array}{l}\text { Protein } \\
\text { (mg/g wet pellet) }\end{array}$} & \multirow{2}{*}{$\begin{array}{l}\text { Catechol- } \\
\text { amines } \\
\text { (mg/g wet } \\
\text { pellet) }\end{array}$} & \multicolumn{2}{|c|}{$\begin{array}{l}\text { g catecholamines } / \mathrm{g} \\
\text { protein }\end{array}$} & \multirow{2}{*}{$\begin{array}{l}\% \text { soluble } \\
\text { protein } \\
\text { (total) }\end{array}$} \\
\hline & Total & Soluble & & Total & Soluble & \\
\hline \multicolumn{7}{|c|}{$\begin{array}{l}\text { Differential centrifugation on } 0.32 \mathrm{M} \text { sucrose } \\
\text { Washed and resuspended in: }\end{array}$} \\
\hline $\begin{array}{l}\text { a. } 0.15 \mathrm{M} \mathrm{K}_{2} \mathrm{SO}_{4} \\
(337 \mathrm{mosM})\end{array}$ & 56.2 & 38.9 & 21.3 & 0.379 & 0.547 & 69.3 \\
\hline $\begin{array}{l}\text { b. } 0.2 \mathrm{M} \mathrm{KCl} \\
(368 \mathrm{mosM})\end{array}$ & 40.4 & 24.1 & 12.5 & 0.309 & 0.519 & 59.6 \\
\hline $\begin{array}{l}\text { c. } 0.16 \mathrm{M} \mathrm{KCl} \\
(293 \mathrm{mosM})\end{array}$ & 44.7 & 35.0 & 15.3 & 0.341 & 0.436 & 78.3 \\
\hline \multicolumn{7}{|c|}{$\begin{array}{l}\text { l'icoll-sucrose }-2 \mathrm{H}_{2} \mathrm{O} \text { gradient } \\
\text { Washed and resuspended in: }\end{array}$} \\
\hline $\begin{array}{l}\text { a. } 0.15 \mathrm{M} \mathrm{K}_{2} \mathrm{SO}_{4} \\
(337 \mathrm{mosM})\end{array}$ & 70.3 & 51.3 & 25.2 & 0.359 & 0.491 & 73.0 \\
\hline $\begin{array}{l}\text { b. } 0.2 \mathrm{M} \mathrm{KCl} \\
(368 \mathrm{mosM})\end{array}$ & 57.7 & 37.6 & 15.9 & 0.275 & 0.423 & 65.2 \\
\hline $\begin{array}{l}\text { c. } 0.16 \mathrm{M} \mathrm{KCl} \\
(293 \mathrm{mosM})\end{array}$ & 57.4 & 35.5 & 14.5 & 0.253 & 0.409 & 61.8 \\
\hline $\begin{array}{l}\text { d. } 0.3 \text { M sucrose } \\
(312 \text { mos } M)\end{array}$ & 69.5 & 44.9 & 24.0 & 0.345 & 0.534 & 64.6 \\
\hline
\end{tabular}


CATECHOLAMINF/PROTEIN AND CATECHOLAMINE/ATP RATIOS DERIVED FROM NMR SIMULATIONS

\begin{tabular}{lll}
\hline Preparation of sample & g catecholamines/g proteins & mol catecholamines/mol ATP \\
\hline $\begin{array}{l}\text { Differential centrifugation in } 0.32 \mathrm{M} \text { sucrose } \\
\text { Washed and resuspended in: }\end{array}$ & \\
a. $0.15 \mathrm{M} \mathrm{K}_{2} \mathrm{SO}_{4}[7]$ & $1.075 \pm 0.043$ & $4.33 \pm 0.633$ \\
b. $0.2 \mathrm{M} \mathrm{KCl}[5]$ & $0.925 \pm 0.092$ & $4.03 \pm 0.293$ \\
c. $0.16 \mathrm{M} \mathrm{KCl} \mathrm{[3]}$ & $0.943 \pm 0.098$ & $4.88 \pm 0.092$ \\
Ficoll-sucrose- ${ }^{2} \mathrm{H}_{2} \mathrm{O}$ gradient & & \\
Washed and resuspended in: & & $3.16 \pm 0.485$ \\
a. $0.15 \mathrm{M} \mathrm{K} \mathrm{SO}_{4}[4]$ & $1.13 \pm 0.199$ & 2.56 \\
b. $0.16 \mathrm{M} \mathrm{KCl}$ & 0.702 & \\
\hline
\end{tabular}

confidence level using asymptotic standard deviations computed by the fitting program.

Catecholamine : protein ratios determined by NMR are systematically larger by about $90 \%$ than the same ratios determined by biochemical assay. This difference indicates that nearly half of the protein freed upon lysis is not NMR visible. This result is discussed in more detail below. Aside from the systematic difference in magnitude, the catecholamine:protein ratios determined by NMR show the same kind of variation among the different preparative procedures as do the ratios determined classically. For example, chromaffin granules prepared on a Ficoll gradient and washed in $0.16 \mathrm{M} \mathrm{KCl}$ are significantly reduced in catecholamine. Granules prepared on the Ficoll gradient and washed in $0.15 \mathrm{M}$ or $0.16 \mathrm{M} \mathrm{K}_{2} \mathrm{SO}_{4}$ do not show this loss.

There appears to be a slight reduction in the ratio for granules prepared by differential centrifugation and washed with $\mathrm{KCl}$, but this may not be significant. Because of apparent catecholamine loss from granules prepared on the Ficoll gradient and washed in $\mathrm{KCl}$, this method was not used subsequently.

The molar ratio of catecholamine:ATP determined by NMR is also shown in Table II. Values obtained for samples prepared by differential centrifugation in $0.32 \mathrm{M}$ sucrose are quite comparable to values obtained from classical assays [2]. Ficoll preparations gave considerably lower ratios, especially the preparation washed in $0.16 \mathrm{M} \mathrm{KCl}$. The ATP peaks ( $\mathrm{H}-2$ and $\mathrm{H}-8)$ are very broad and essentially unresolved in all the Ficoll preparations, however (see above and Fig. 2), and the assignment of accurate linewidths and chemical shifts was not possible. For this reason we attach little significance to the low catecholamine: ATP ratios found in the Ficoll preparations. Of course a somewhat lower value would be expected in the $0.16 \mathrm{M} \mathrm{KCl}$-washed sample, for which catecholamine loss is evident.

Table III contains a comparison of the protein and catecholamine concentrations obtained by the two methods. It is readily seen that the catecholamine concentrations obtained by both methods are in approximate agreement while the catecholamine:protein ratios are significantly different. In all cases, between $40 \%$ and $50 \%$ of the protein found in the soluble fraction on lysing the chromaffin granules is not seen by NMR. Presumably protein in the soluble 
TABLE III

COMPARISON OF BIOCHEMICAL AND NMR ASSAYS OF THE CATECHOLAMINE CONCENTRATION, AND OF THE CATECHOLAMINE/PROTEIN RATIOS

\begin{tabular}{|c|c|c|c|c|}
\hline \multirow[t]{3}{*}{ Preparation of sample } & \multirow{2}{*}{\multicolumn{2}{|c|}{$\begin{array}{l}\text { Catecholamine concentration } \\
\text { (mg/g wet pellet) }\end{array}$}} & \multicolumn{2}{|c|}{ g catecholamines/g protein } \\
\hline & & & \multirow{2}{*}{ NMR } & \multirow{2}{*}{ Classical } \\
\hline & NMR & Classical & & \\
\hline \multicolumn{5}{|c|}{$\begin{array}{l}\text { Differential centrifugation in } 0.32 \mathrm{M} \text { sucrose } \\
\text { Washed and resuspended in: }\end{array}$} \\
\hline a. $0.15 \mathrm{M} \mathrm{K}_{2} \mathrm{SO}_{4}$ & 34.1 & 34.1 & 1.075 & 0.566 \\
\hline b. $0.2 \mathrm{M} \mathrm{KCl}$ & 27.2 & 31.3 & 0.925 & 0.538 \\
\hline c. $0.16 \mathrm{M} \mathrm{KCl}$ & 21.4 & 19.3 & 0.943 & 0.484 \\
\hline \multicolumn{5}{|c|}{$\begin{array}{l}\text { Ficoll-sucrose }{ }^{2} \mathrm{H}_{2} \mathrm{O} \text { gradient } \\
\text { Washed and resuspended in: }\end{array}$} \\
\hline a. $0.15 \mathrm{M} \mathrm{K}_{2} \mathrm{SO}_{4}$ & 19.1 & 21.7 & 1.13 & 0.509 \\
\hline
\end{tabular}

phase is present in two very different forms, one much more highly mobile than the other. The possibility that chromogranin is present in intact chromaffin granules as a precipitate that dissolves upon lysis was checked by observing the NMR spectrum of granules lysed in 40 vols. of cold ${ }^{2} \mathrm{H}_{2} \mathrm{O}$. The computer simulation of the lysate gave a catecholamine:protein ratio which was even higher than the value from intact granules (Table IV). The catecholamine present in the lysate was assayed and found to equal the catecholamine concentration observed via NMR. Apparently, lysing in large volumes of ${ }^{2} \mathrm{H}_{2} \mathrm{O}$ had converted a portion of the NMR-visible protein into an NMR-invisible form, possibly due to aggregation at the lowered ionic strength. Clearly, however, the soluble protein was not NMR invisible simply due to limited solubility in intact chromaffin granules. Addition of $8 \mathrm{M}$ urea did not substantially decrease the catecholamine:protein ratio determined by NMR. The lysate was then hydrolysed in $6 \mathrm{~N} \mathrm{HCl}\left(110^{\circ} \mathrm{C}, 20 \mathrm{~h}\right)$. The integrated intensity in the spectral region containing resonances from methylene and methyl protons of amino acid side chains was measured before and after hydrolysis. A reference peak of TSS at known concentration was included in the sample to correct for instrumental variations in measured intensities. Upon hydrolysis the intensity increased by a factor of 1.74 , confirming the presence of a substantial quantity of NMRinvisible protein in the original lysate.

TABLE IV

CATECHOLAMINE:PROTEIN RATIO OF LYSATE AND HYDROLYZED LYSATE

Chromaffin granules suspended in $0.16 \mathrm{M} \mathrm{K}_{2} \mathrm{SO}_{4}$ (g catecholamines/g protein)

Classical assay

Soluble lysate (3) $\quad 0.579$

NMR assay

Soluble lysate (2) $\quad 1.43$

Hydrolyzed lysate (1) $\quad 0.82$ 


\section{Discussion}

The data in Table III show that approximately 97\% (averaged over all preparations) of the total catecholamines are present in the NMR-visible aqueous phase. The NMR determination of catecholamine concentration was obtained from the ratio of integrated intensities of the catecholamine aromatic peak to the methyl peak of an NMR standard, TSS, added to a known concentration. Intensity contributions due to overlapping protein resonances were of the order of $15 \%$ in both the aromatic and TSS regions. The intensity ratio was corrected for these contributions by using the spectral simulation program. The corrected ratio contains an estimated uncertainty of $\pm 7 \%$ which is due in part to the possibility of slight baseline drift and phase error. Deviations between the total and NMR-visible catecholamine concentrations have an average value of $8.0 \%$, which is consistent with the estimated uncertainties in the NMR and biochemical measurements. Concentrations obtained from NMR are not systematically lower than the overall concentrations as would be expected if an appreciable immobile catecholamine fraction were present. The average deviation provides an upper estimate of the bound catecholamine fraction.

It should be emphasized that the NMR data do not exclude the presence of relatively low molecular weight complexes between epinephrine and ATP. Very labile complexes with a $3: 1$ (epinephrine:ATP) ratio have been observed in aqueous solutions at $\mathrm{pH} 5.6$ [38], which is probably the intragranular $\mathrm{pH}$ [11, $14,39,40]$. A more recent proton NMR study [41] has concluded that the actual stoichiometry is lower, $1: 1$ or 2:1. Somewhat larger aggregates have been found in the presence of $20-50 \mathrm{~mol} \% \mathrm{Ca}^{2+}$ and $\mathrm{Mg}^{2+}$ [42-44]. Such complexation produces line broadening that is described roughly by the StokesEinstein relation [45], $\Delta \nu_{1 / 2} \alpha \tau_{\mathrm{R}} \approx 4 \pi a^{3} \eta / 3 k T$, where $\Delta \nu_{1 / 2}$ is the NMR linewidth, $\tau_{\mathrm{R}}$ is the rotational correlation time, $a$ is the radius of the aggregate, and $\eta$ is bulk viscosity. Thus the linewidth increases as the volume of the complex times $(\pi / k T)$. Relatively low molecular weight aggregates would be consistent with the $15-40-\mathrm{Hz}$ linewidths seen in the proton spectra. We have pointed out previously however, that such complexes appear to be inconsistent with $\mathrm{T}_{1}$ and NOE measurements [9].

Concentration ratios of catecholamine:ATP in the NMR-visible phase contain error limited by the integrals of the $\mathrm{H}_{2}$ and $\mathrm{H}_{8}$ resonances of ATP. Since these peaks are relatively weak and broad, the measured ATP concentration contains substantially greater uncertainty than does the value for catecholamines. The non-linear least-squares fitting program computes an asymptotic standard deviation to the ATP:catecholamine ratio. Error limits at the 95\% confidence level were computed and are given in Table II; limits are generally smaller for the media that gave narrower lines. Winkler [2] in his survey has given an average value of 4.5 for the overall catecholamine:ATP mol ratio obtained by four groups. NMR ratios calculated from samples prepared by differential centrifugation are within $8 \%$ of this value indicating that most (and possibly all) of the ATP is present with catecholamine in the aqueous phase. Values obtained from the Ficoll preparations are substantially lower but are of doubtful quantitative significance (see above). 


\section{The state of the soluble protein}

The fact that chromogranin gives resolvable structure in the proton and C-13 spectra [6-9] is a direct consequence of the predominantly random-coil structure of these proteins and the high side chain and segmental mobility. It is shown in Tables III and IV that approximately 55\% of the protein freed upon lysis is in an NMR-visible form. Presumably the NMR-invisible fraction is present in one of the following states: (1) globular protein (e.g. DBH); (2) protein associated with high molecular weight soluble complexes, such as the soluble lipoprotein complexes isolated by Hogue-Angelletti and Sheetz [15], or (3) soluble chromogranin that is present in a precipitated form. From the extremely high solubility of purified chromogranin it seems likely that most of this material would be dissolved in the aqueous phase. Reconstituted solutions containing epinephrine, ATP and chromogranin dissolved at physiological concentrations have been prepared $[7,40]$. If precipitated soluble protein were present in the intact granules, it would be expected to appear in spectra of the unconcentrated lysate. This did not occur, and even the addition of $8 \mathrm{M}$ urea did not substantially increase the NMR-visible protein. While it has been reported that native chromogranin has some secondary structure [46], it appears that this kind of structure cannot substantially account for the observed loss of NMR intensity. Recovery of most of this intensity was obtained only after complete hydrolysis.

Certainly the amount of soluble DBH in the aqueous phase (about $5 \%$ by weight [2]) cannot account for the entire NMR-invisible protein fraction. On the other hand, the lipoprotein complexes isolated by Hogue-Angelletti and Sheetz [15] may be relevant, although they have not yet been characterized extensively. Complexes with molecular weights of several hundred thousand would be highly broadened. It is also interesting that these complexes are stable under highly denaturing conditions (guanidine hydrochloride), as is the NMRinvisible protein. The total protein content of the various complexes found has not been reported however, and may be too small to account for the entire NMR-invisible fraction.

\section{Bound catecholamines}

The intensity measurements described above demonstrate that nearly all catecholamine and ATP are present in the highly mobile aqueous phase. From the known structure and composition of chromaffin granules, however, one would expect an important, though possibly quite small, fraction of catecholamine to exist in a state of greatly restricted mobility. It has been reported that catecholamine binds to the chromaffin granule membrane [47]. Two types of site have been reported. One of these has low capacity, high affinity and is reserpine sensitive (possibly a carrier molecule). The other has relatively high capacity and low affinity. It has been suggested that catecholamine in its uncharged form partitions into the lipid phase $[39,47]$ although this point is controversial [17]. Catecholamines may also be bound by intragranular dopamine $\beta$-hydroxylase.

We have not obtained direct evidence for labile catecholamine binding, but one observation would be consistent with this hypothesis. This is the dramatic selective broadening and/or shift in the $\beta-\mathrm{CH}_{2}$ catecholamine resonance of chro- 
maffin granules that are suspended in $0.16 \mathrm{M} \mathrm{K}_{2} \mathrm{SO}_{4}$ (Fig. 3). The only explanation for this effect that has occurred to us is labile binding of a small portion of catecholamine to dopamine $\beta$-hydroxylase. Broadening would result only if the bound fraction, which could be very small (less than $10^{-3}$ ), were in rapid equilibrium with the aqueous pool. The fact that broadening is selective at the $\alpha-\mathrm{CH}_{2}$ resonance requires a specific magnetic perturbation at these nuclei. This would occur if these hydrogens were close to the pair of $\mathrm{Cu}(\mathrm{II})$ ions at the active site. Structural analogues of the catecholamines competetively inhibit DBH activity, although the binding is apparently relatively nonspecific [48, 49]. In general, paramagnetic line broadening will depend on the bound fraction, on the structure at the active site, and on the kinetic parameters of the chemical exchange reaction [50]. It is surprising that although the effect was observed repeatedly, it was pronounced only in the $0.16 \mathrm{M} \mathrm{K}_{2} \mathrm{SO}_{4}$ medium. The mechanism by which the external medium influences line broadening is unknown.

\section{Conclusions}

The fact that high concentrations of epinephrine and ATP (approximately $700 \mathrm{mM}$ total) are dissolved in the aqueous phase has implications for hypotheses concerning the relatively low osmolality of the internal medium. A structured phase containing a substantial fraction of osmotically inactive catecholamine is not consistent with the measured composition of the aqueous phase. The osmotic activity of dissolved catecholamines must be lowered substantially by intermolecular interactions in the solution phase. Furthermore, such interactions must be consistent with quite short rotational correlation times that have been reported elsewhere. Relevant interactions could result either from direct molecular complexation between catecholamines and ATP, or from electrostatic interactions between low molecular weight ions and chromogranin, which is present as a random-coil polyelectrolyte. Further studies are needed to clarify the nature of these interactions.

\section{Acknowledgements}

This research was supported in part by grants from the Michigan Heart Association and by Institutional Research Grant No. In-40 P to the University of Michigan from the American Cancer Society. We acknowledge very helpful discussions with Professor R.W. Holz. R.S. received financial support in the form of a Rackham fellowship.

\section{References}

1 Carlsson, A. and Hiliarp, N.-A. (1958) Acta Phy siol. Scand. 44, 163-169

2 Winkler, H. (1976) Neuroscience 1, 65-80

3 Dreyfus, H., Aunis, D., Harth, S. and Mandel, P. (1977) Biochim. Biophys. Acta 489, $89-97$

4 Geissler, D. Martinek, A., Margolis, R.U., Margolis, R.K., Skrivanek, J.A., Ledeen, R., König. P. and Winkler, H. (1977) Neuroscience 2, 685-693

5 Blaschke, E., Bergqvist, U, and Uvnas, B. (1976) Acta Physiol. Scand. 97, 110-120

6 Daniels, A.J., Korda, A., Tanswell, P., Williams, A. and Williams, R.d.P. (1974) Proc. R. Soc. (Lond.) B 187, 353-361 
7 Daniels, A.J., Williams, R.J.P. and Wright, P.E. (1978) Neuroscience 3, 573-585; (1976) Nature 261. 321-322

8 Sharp, R.R. and Richards, E.P. (1977) Biochim. Biophys. Acta 497, 14-28

9 Sharp, R.R. and Richards, E.P. (1977) Biochim. Biophys. Acta 497, 260-271

10 Sharp, R.R. and Sen, R. (1978) Biochim. Biophys. Acta 538, 155-163

11 Casey, R.P., Njus, D., Radda, G.K. and Sehr, P.A. (1977) Biochemistry 16, 972-977

12 Morris, S.J, and Schovanka, I. (1977) Biochim. Biophys. Acta 464, 53-64

13 Morris, S.J., Schultens, H.A. and Schober, R. (1977) Biophys. J. 20, 33-48

14 Johnson, R.G. and Scarpa, A. (1976) J. Gen. Physiol. 68, 601-631

15 Hogue-Angelletti, R.A. and Sheetz, P. (1978) J. Biol. Chem. 253, 5613-5616

16 Hillarp, N.-A. (1959) Acta Physiol. Scand. 47, 271-279

17 Njus, D. and Radda, G.K. (1978) Biochim. Biophys. Acta 463, 219-244

18 Stjärne, L. (1972) in Catecholamines, Handbook of Experimental Pharmacology, Vol. 33, pp. 231269, Springer-Verlag, Berlin

19 Smith, A.D. (1968) in Intexactions of Drugs and Subcellular Components in Mammalian Cells (Camp. bell, P.N., ed.), pp. 239-292, Churchill, London

20 Granot, J, and Rosenheck, K, (1978) FEBS Lett. 95, 45-48

21 Smythies, J.R., Antun, F., Yank, G. and Yorke, C. (1971) Nature 231, 185-188

22 Berneis, K.H., Pletcher, A. and DaPrada, M. (1970) Br. J. Pharmacol. 39, 382-389; (1971) Experientia $27,917-918$

23 Helle, K.B. and Serck-Hanssen, G. (1973) Frontiers in Catecholamine Research, pp. 467-469, Pergamon, London; (1975) Mol. Cell. Biochem, 6, 127-146

24 Slotkin, T.A., Ferris, R.M. and Kirshner, N. (1971) Mol. Pharmacol. 7, 308-316 -

25 Slotkin, T.A. and Kirshner, N. (1971) Mol. Pharmacol. 7, 581-592

26 Hillarp, N.-A. (1960) Acta Physiol. Scand. 50, 8-22

27 Taugnex, G. and Hasselbach, W. (1966) Naunyn-Schmiedeberg's Arch. Exp. Pathol. Pharmakol. 255 $266-286$

28 Slotkin, T.A. and Kirshner, N. (1973) Biochem. Pharmacol. 22, 2492-2497

29 Emsley, J.W., Feeney, J. and Sutcliff. L.H. (1967) High Resolution Nuclear Magnetic Resonance Spec. troscopy, Chapter 9, Pergamon, Oxford

30 Bartlett, S.F. and Smith, A.D. (1974) Methods Enzymol, 31, 379-389

31 Trifaro, J.M. and Dworkind, J. (1970) Anal. Biochem. 34, 403-409

32 Kirshner, N. (1962) J. Biol. Chem. 237, 2311-2317

33 Lowry, O.H., Rosebrough, N.J., Farr, A.L. and Randall, P. (1951) J. Biol. Chem. 193, 265-275

34 Bradford, M.M. (1976) Anal. Biochem. 72, 248-254

35 Weil-Malherbe, H. (1973) in Methods of Biochemical Analysis (Glick, D., ed.), Suppl. Vol., pp. 119152 Interscience, New York

36 BMDX85-Non-linear Least Squares, Statistical Research Laboratory, The University of Michigan, Ann Arbor, MI 48109

37 Green, D.J., Westhead, E.W., Langley, K.H. and Satelle, D.B. (1978) Biochim. Biophys. Acta 539, $364-371$

38 Weiner, N. and Jardetzky, O. (1964) Naunyn-Schmiedeberg's Arch. Exp. Pathol. Pharmakol. 248, $308-318$

39 Johnson, R.G., Carlson, N.J, and Scarpa, A. (1978) J. Biol. Chem. 253, 1512-1521

40 Njus, D., Sehr, P.A., Radda, G.K., Ritchie, G.A. and Seeley, P.J. (1978) Biochemistry 17, 43374343

41 Granot. J. (1978) J. Am. Chem. Soc. 100, 2886-2890; ibid., 1539-1548

42 Berneis, K.H., Pletscher, A. and DaPrada, M. (1969) Nature 224, 281-283

43 Pai, V.S. and Maynert, E.W. (1972) Mol. Pharmacol. 8, 82-87

44 Tuck, L.D. and Baker, J.K. (1973) Chem. Biol. Interactions 7, 335-366

45 Abragam, A. (1961) The Principles of Nuclear Magnetism, p. 300, Oxford

46 Kirshner, A.G. and Kirshner, N. (1969) Biochim. Biophys. Acta 181, 219-225

47 Nichols, J.W. and Deamer, D.W. (1976) Biochim. Biophys. Acta 455, 269-271

48 Goldstein, M., Joh, T.H. and Garvey, T.Q. (1968) Biochemistry 7, 2724-2730

49 Creveling, C.R., Daly, J.W., Witkop, B, and Udenfriend, S. (1962) Biochim. Biophys. Acta 64, 125134

50 Dwek, R.A. (1973) NMR in Biochemistry, Chapter 9, Clarendon, Oxford 